\title{
Sven Bernecker
}

\section{Reinholds linguistischer Schematismus}

Karl Leonhard Reinholds Elementarphilosophie ist das Verbindungsstück zwischen Kants Kritischer Philosophie und den idealistischen Systementwürfen des jungen Fichte und Schelling. Reinhold war der Auffassung, Kant habe die Resultate gegeben, zu denen noch die Prämissen gefunden werden müssen. Die Elementarphilosophie tritt mit dem Anspruch auf, die Prämissen zur kantischen Vernunftkritik zu liefern, indem ein oberster Grundsatz des Bewusstseins angegeben wird, aus dem die kantischen Theoreme abgeleitet werden können.

Reinhold hat die Elementarphilosophie nur sieben Jahre lang vertreten. Im Jahr 1796 erklärt er sich öffentlich zum Verfechter von Fichtes Wissenschaftslehre. Zwei Jahre später distanziert er sich von der Wissenschaftslehre und vertritt einige Monate lang einen Zwischenstandpunkt zwischen Fichtes Wissenschaftslehre und der Glaubensphilosophie von Friedrich Heinrich Jacobi. Danach ist Reinhold zwölf Jahre lang Verfechter des von Christoph Gottfried Bardili propagierten Rationalen Realismus. In den letzten elf Jahren seines Lebens entwickelt Reinhold dann wieder ein eigenständiges - und zwar sprachphilosophisches - System.

In diesem Aufsatz stelle ich eine neue Interpretation der Reinhold'schen Sprachphilosophie vor. Mein Ziel ist es zu erklären, wie Reinhold der Meinung sein konnte, seine Sprachphilosophie stelle, ebenso wie seine Elementarphilosophie, den Versuch dar, Kants Kritische Philosophie zu fundieren. Außerdem möchte ich zeigen, worin die philosophische Bedeutung von Reinholds Ansatz gegenüber den Sprachphilosophien seiner Zeitgenossen besteht.

\section{Die Interdependenz von Sprache und Denken}

Kant hält bekanntlich die Sprache für ein bloßes Organ für die Formulierung der sprachvorgängigen Gedanken. Die Vernunft drückt sich durch die Sprache aus, aber die Sprache beeinflusst nicht das Denken. Kants instrumentalistische Sprachauffassung und der von ihm behauptete Primat des Denkens vor dem Sprechen kommt beispielsweise in seiner Anthropologie-Schrift zum Ausdruck, wo es lapidar heißt: „Alle Sprache ist Bezeichnung der Gedanken.“1 Reinhold vertritt

1 Kant: Anth, AA 07: 192. 29.

Sven Bernecker, [Adresse] 
demgegenüber die Ansicht, die Sprache sei nicht bloß Ausdruck, sondern auch Bedingung der Möglichkeit des Denkens. Und weil die Sprache konstitutiv für das Denken ist, geht von einem unreflektierten (partikularen) Sprachgebrauch für die Philosophie Gefahr aus. Aus diesem Grund hält Reinhold die Sprachanalyse für eine unumgängliche Propädeutik der Philosophie.

Reinhold fordert eine „Critik der Sprache“, welche die Aufgabe hat zu zeigen, wie die

Wandelbarkeit und Vieldeutigkeit des Sprachgebrauchs, durch welche die Wörter, welche dem Denken dienen sollten, dasselbe beherrschen, und die Vernunft in die ihrem Werkzeuge, der Sprache eigenthümliche Wandelbarkeit und Dienstbarkeit hineinziehen. ${ }^{2}$

Obwohl Reinhold einen allgemeinen und verbindlichen Sprachgebrauch für möglich hält, wäre es ein Fehler, seine Sprachkritik mit dem Idealsprachenprogramm, wie es von Frege, Russell und Carnap verfolgt wird, in Verbindung zu bringen. Reinholds Sprachanalyse zielt nicht auf eine formale Kunstsprache ab. Seine Sprachkritik versteht sich als eine „Dollmetscherin“, die die „ursprünglichen und wechsellosen Bedeutungen“3 der natürlichen Sprache aufdeckt. Außerdem unterscheidet sich Reinholds Sprachkritik von dem Idealsprachenprogram dadurch, dass er nicht die Absicht hat, die spekulative Philosophie zu eliminieren. Statt die Philosophie durch die Naturwissenschaft ersetzen zu wollen, möchte Reinhold der Philosophie ein sprachkritisch gereinigtes Fundament geben. Die Philosophie soll neu begründet, nicht ersetzt werden.

Die These von der wechselseitigen Abhängigkeit von Sprache und Denken ist natürlich nicht Reinholds Entdeckung, sondern sie findet sich bereits bei Johann Georg Hamann, Johann Gottfried Herder, Wilhelm von Humboldt und Johann Georg Sulzer. Reinhold ist auch nicht der erste, der den Gedanken von der gegenseitigen Abhängigkeit von Denken und Sprechen in kritischer Wendung gegen Kants Annahme der Unabhängigkeit der Vernunft von der Sprache entwickelt. Hamann, Herder und Jacobi haben schon vor Reinhold Kant den Vorwurf der Sprachverdrängung oder Sprachvergessenheit gemacht. Das Neue an Reinholds These von der wechselseitigen Abhängigkeit von Denken und Sprache ist die Art und Weise, wie sie begründet wird. Statt die Abhängigkeitsthese von außen an die Transzendentalphilosophie heranzutragen, bettet Reinhold sie in die Transzendentalphilosophie ein. Während Hamann und Herder der Auffassung sind, der mentalistische Ansatz Kants sei prinzipiell nicht in der Lage, der Bedeutung der

2 Reinhold, Karl Leonhard: Grundlegung einer Synonymik für den philosophischen Sprachgebrauch in den philosophischen Wissenschaften. Kiel 1812, VIII.

3 Reinhold: Grundlegung einer Synonymik, 37. 
Sprache für das Denken Rechnung zu tragen, zeigt Reinhold, wie der Sprache innerhalb der Transzendentalphilosophie ein Ort zugewiesen werden kann. Reinhold macht die Sprachphilosophie zu einem integrativen Bestandteil der Transzendentalphilosophie.

\section{Die Vermittlung von Sinnlichkeit und Verstand}

Eine Kernthese der Kritik der reinen Vernunft besagt, Erfahrung sei ein Produkt von Sinnesdaten und Verstandesprinzipien, von Anschauungen und Begriffen, von Rezeptivität und Spontaneität. Das Postulat des Dualismus der Erkenntnisquellen ist entscheidend für Kants Lösung des Streits zwischen dem Empirismus und dem Rationalismus. Aus dem Dualismus der Erkenntniskräfte resultiert für Kant aber auch ein Problem. Da uns ohne Sinnlichkeit kein Gegenstand gegeben ist und ohne Verstand keiner gedacht werden kann, müssen sich beide Erkenntnisquellen verbinden. ${ }^{4}$ Aber wie können sich die zwar als gleichberechtigt, aber gleichzeitig strikt voneinander geschiedenen Erkenntniskräfte Sinnlichkeit und Verstand miteinander verbinden, sodass Erfahrung entsteht? Kant nimmt im Verlauf des kritischen Geschäfts zwei Anläufe dieses Problem zu lösen: in der „Transzendentalen Deduktion der reinen Verstandesbegriffe“ und im Schematismus-Kapitel.

Reinhold ist der Auffassung, dass durch die Aufwertung der Sprache in den Rang einer transzendentalen Bedingung des Denkens ein Grundproblem der kantischen Philosophie - die Vermittlung zwischen Sinnlichkeit und Verstand gelöst werden kann. Nur durch die Sprache können Sinnlichkeit und Verstand so miteinander vermittelt werden, dass Erfahrung möglich ist. Reinhold erklärt:

\footnotetext{
Und dennoch ist es unläugbar die Sprache, und nur sie, wodurch der, dem menschlichen Erkenntnißvermögen eigene, und wesentliche, Zusammenhang zwischen der Sinnlichkeit und dem Denkvermögen im Menschen zunächst vermittelt wird. Die Sprache gehört diesen beyden Vermögen an, indem Sie dem Einen das an ihr Sinnenfällige, das Hörbare der articulirten Laute und das Sichtbare der Schriftzeichen, dem Anderen aber was durch ihr Sinnenfälliges nur bezeichnet wird, die Begriffe welche durch die einzelnen Wörter, die Urtheile welche durch einzelne Sätze, und die Schlüsse welche durch verbundene Sätze ausgesprochen werden, verdankt. ${ }^{5}$
}

4 Kant: KrV, A 51/B 75.

5 Reinhold, Karl Leonhard: Das menschliche Erkenntnisvermögen aus dem Gesichtspunkte des durch die Wortsprache vermittelten Zusammenhangs zwischen der Sinnlichkeit und dem Denkvermögen. Kiel 1816, $3 \mathrm{f}$. 
Reinhold ist also der Ansicht, die Sprache habe transzendentalen Charakter, weil Erfahrung das Zusammenspiel von Sinnlichkeit und Verstand erfordert und nur die Sprache zwischen Sinnlichkeit und Verstand vermitteln kann. Um zu verstehen, weshalb Reinhold meint, die Sprache könne zwischen den beiden Erkenntnisquellen Sinnlichkeit und Verstand vermitteln, müssen wir uns Kants Schematismuslehre in Erinnerung rufen.

\section{Kants schematische Hypotypose}

Im Schematismus-Kapitel der Kritik der reinen Vernunft zeigt Kant, wie die Kategorien auf die in der Anschauung gegebenen Sinnesdaten angewendet werden. Das Schema ist ein „Produkt“ der produktiven Einbildungskraft. ${ }^{6}$ Die produktive Einbildungskraft ist die Fähigkeit, mentale Bilder (figürliche Vorstellungen) von Dingen zu produzieren, die wir nicht gegenwärtig wahrnehmen. Da das, was die Einbildungskraft normalerweise produziert, mentale Bilder sind, unterscheidet Kant Schemata von mentalen Bildern. Der Unterschied besteht darin, dass das Schema eine Regel des Verstandes für die Einbildungskraft ist, damit die Einbildungskraft mentale Bilder produzieren kann. Das Schema ist „eine Regel der Bestimmung unserer Anschauung, gemäß einem gewissen allgemeinen Begriff“. 7

Schemata sind Kant zufolge das vermittelnde Dritte zwischen dem Verstand und der Sinnlichkeit. Sie sind intellektuell und sinnlich zugleich. Schemata sind intellektuell, da sie Regeln des Verstandes sind; und sie sind sinnlich, da diese Regeln Bauanleitungen oder Schablonen für mentale Bilder liefern. Kant erläutert den Unterschied zwischen Bild und Schema unter anderem anhand des Beispiels des rein sinnlichen Begriffs Dreieck. In welcher Beziehung steht der rein sinnliche Begriff des Dreiecks zu konkreten Dreiecken ${ }^{8}$ Dem Begriff des Dreiecks kann kein Bild in der Einbildungskraft adäquat sein, da das Bild notwendigerweise ein rechtwinkliges oder schiefwinkliges Dreieck darstellt, der Begriff des Dreiecks aber wegen seiner Allgemeinheit nicht auf eine dieser Arten von Dreiecken eingeschränkt werden kann. Kant zieht daraus die Konsequenz, dass sich der Begriff des Dreiecks nicht auf Bilder, sondern auf Schemata bezieht. Diese Schemata sind die Regeln für die Einbildungskraft zur Produktion reiner Gestalten im Raume. ${ }^{9}$

6 Kant: KrV, A 140/B 179.

7 Kant: KrV, A 141/B 180.

8 Kant: KrV, A 140f./B 180.

9 Kant: KrV, A 141/B 180. 


\section{Reinholds sprachliche Hypotypose}

Üblicherweise wird das Schematismus-Problem als das der Anwendung eines allgemeinen Begriffs auf ein partikulares Bild verstanden. Das Problem ist dann dies: Wie kann die Einbildungskraft für einen allgemeinen Begriff - sei es ein empirischer Begriff, ein rein sinnlicher Begriff oder ein reiner Verstandesbegriff die passenden sich auf Einzeldinge beziehenden mentalen Bilder konstruieren?

Reinhold interpretiert die Schematismus-Lehre unter einem anderen Aspekt als dem der Umfangseinschränkung. Er fragt: Warum exemplizifiert ein mentales Bild, beispielsweise das eines gleichschenklichen Dreiecks den Begriff Dreieck? Damit ein Bild einen Begriff explifizieren kann, muss sein repräsentationaler Gehalt mit dem des Begriffes (zumindest teilweise) übereinstimmen. Aber woher bekommt ein mentales Bilder seinen repräsentationalen Gehalt? Reinhold zufolge erhalten mentale Bilder ihren repräsentationalen Gehalt durch die Sprache. Ohne die Sprache wären Bilder bedeutungslos und könnten deshalb nicht zur Versinnlichung der Begriffe herangezogen werden.

In der Schrift Das menschliche Erkenntnisvermögen (Kiel 1816) setzt sich Reinhold ausdrücklich mit Kants Schematismus-Lehre auseinander. Er unterscheidet zwischen „Begriffen“ und „Worten“ (die er auch „Denkbilder“ nennt), „Einzelbildern“ (Portraits) und „Schemata“ (die er auch „Gemeinbilder“ nennt). Allerdings verwendet Reinhold den Terminus „Schema“ etwas anders als Kant. Das Schema ist bei Reinhold ein mentales Bild und nicht, wie bei Kant, eine Regel zur Erzeugung solcher Bilder.

Reinholds konstruktive Kant-Kritik besagt nun, dass die Vermittlung zwischen Sinnlichkeit und Verstand nur mit Hilfe der Sprache geleistet werden kann. Die Subsumtion des Empfindungsmaterials unter empirische und rein sinnliche Begriffe erfolgt nicht allein mit Hilfe von Schemata, sondern auch mit Hilfe von Worten oder genauer gesagt mit Hilfe allgemeiner Terme. Die Schemata (Gemeinbilder) können nur in Verbund mit den Worten (Denkbildern) zwischen der Sinnlichkeit und dem Verstand vermitteln. Reinhold erklärt:

Das Gemeinbild würde sich freylich [...] nicht ohne das Denkvermögen, und ohne das, dem menschlichen Denkvermögen nothwendige Wort, (das Denkbild) einfinden können. Aber das Gemeinbild ist keineswegs das Denkbild, und dieses nicht jenes. Beyde werden durch ganz verschiedene Funktionen der, dem Denkvermögen dienenden Einbildungskraft aufgestellt. Beyde sind durch die Einbildungskraft vermittelte Erscheinungen [...] und zwar vergegenwärtigende, repräsentirende, Erscheinungen. Aber durch die Gemeinbilder werden nur wieder andere, ihnen ähnliche, Erscheinungen, in ihrer Abwesenheit, vergegenwärtiget, durch die Wörter aber, in ihrem Unterschiede von den Gemeinbildern, wird von den Er- 
scheinungen, als solchen, hinweggewiesen, und auf das, denselben zum Grunde liegende, unveränderliche Seyn an sich hingewiesen. ${ }^{10}$

Reinhold ist also der Auffassung, die Einbildungskraft produziere neben Schemata (Gemeinbildern) auch Worte (Denkbilder). Den Worten kommt ein Primat zu. Die Einbildungskraft kann nämlich keine Schemata produzieren, wenn sie nicht bereits über Worte verfügt. Sie ist bei der Konstruktion von Schemata auf die Sprache, auf Worte, angewiesen. Der Grund ist, dass die Verstandesregeln, die die Bauanleitungen für Schemata enthalten, sprachlicher Natur sind.

Die Frage, die sich Reinhold stellt, ist also diese: Wie können mentale Bilder repräsentieren? Die unbegriffliche und passive Sinnlichkeit kann für sich alleine keine repräsentierenden Bilder produzieren. Dazu bedarf es der spontanen Leistung des Verstandes. Damit Bilder repräsentieren können, muss es ein Repräsentationssystem geben. Reinhold meint, dieses Repräsentationssystem sei die Sprache. Ohne die Sprache bestehen zwischen Bildern und den bezeichneten Gegenständen höchstens Ähnlichkeitsbeziehungen, aber keine repräsentationalen Beziehungen. Ohne Sprache haben die Bilder keine semantischen Eigenschaften, sie repräsentieren nichts. Reinhold erklärt: „Eine wortlose, gleichwohl aber [...] Begriffe ankündigende Gebehrdensprache, z. B. bey der Pantomimik, und beym Unterrichte der Taubstummen, ist nur unter der Bedingung der bereits schon vorhandenen Wortsprache möglich. “11 Damit also aus den Gebärden Symbole werden, die semantischen Gehalt haben, bedarf es der Wortsprache. Ohne das sprachliche Repräsentationssystem wären die Gebärden sinnlos. Und was für Gebärden gilt, das gilt auch für mentale Bilder. Ein mentales Bild erhält seinen semantischen Gehalt dadurch das Repräsentationssystem der Sprache. Auf Kants Zirkel-Beispiel im Schematismus-Kapitel Bezug nehmend schreibt Reinhold: „Das Bild der Cirkelfigur, z. B. welches das Wort Cirkel begleitet, [...] ist ein Gemeinbild (Schema), welches nur das Aehnliche sinnenfälliger, veränderlicher u. s. w. Cirkelfiguren enthält. “12 Und er fährt fort, dass das Gemeinbild durch das Wort „Zirkel“ Bedeutung erhält.

Man fühlt sich hier natürlich unweigerlich an $\S \S 139 \mathrm{ff}$. von Wittgensteins Philosophischen Untersuchungen erinnert. ${ }^{13}$ Dort stellt sich Wittgenstein die Frage, was es heißt, ein Wort zu verstehen. Eine mögliche Antwort ist es zu sagen, die Bedeutung eines Wortes bestünde in einem mentalen Bild. Wittgenstein verwirft diese Möglichkeit, weil Bilder für sich genommen nichts repräsentieren. Bilder

10 Reinhold: Das menschliche Erkenntnisvermögen, 226.

11 Reinhold: Das menschliche Erkenntnisvermögen, 75, vgl. 84.

12 Reinhold: Das menschliche Erkenntnisvermögen, 88f.

13 Wittgenstein, Ludwig: Philosophische Untersuchungen. Frankfurt am Main 1971. 
können dazu benutzt werden, dieses oder jenes zu repräsentieren, aber sie haben keine intrinsische Bedeutung. Wittgenstein veranschaulicht diesen Gedanken anhand des Piktogramms eines Mannes, der einen Berg hochgeht.

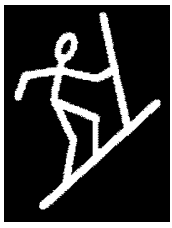

Abb. 1

Das Bild selbst legt nicht fest, was repräsentiert wird: ein Mann, der den Berg hochgeht, oder ein Mann, der den Berg herunterrutscht, oder etwas ganz anderes. Und es hilft auch nichts, wenn man einen Pfeil in das Bild einfügt. Wittgenstein zieht daraus den Schluss, dass Wort- und Zeichenbedeutungen nicht auf intrinsischen Eigenschaften beruhen, sondern auf extrinsischen Eigenschaften, nämlich der Verwendung der Worte beziehungsweise Zeichen durch die Sprechergemeinschaft. Reinhold ist, im Gegensatz zu Wittgenstein, kein semantischer Externalist. Aber er antizipiert Wittgensteins Einsicht, dass Bilder für sich genommen bedeutungslos sind. Reinhold meint, dass nur die Sprache mentalen Bildern Bedeutung geben kann.

Aber ist es in der Tat so, dass Bilder ohne die Sprache gehaltslos wären? Wahrscheinlich gibt es auch andere Repräsentationssysteme als das der Sprache. Denn wie sonst könnte ein Kind eine Sprache erlernen? Jedoch scheinen mir Reinhold und Wittgenstein Recht zu haben, wenn sie betonen, dass ein mentales Bild aus sich heraus nichts repräsentiert. Die Sprache ist vielleicht nicht das einzige Repräsentationssystem, aber sie ist zweifelsohne ein wichtiges Repräsentationssystem.

Die Schemata genannten Regeln des Verstandes zur Versinnlichung der Begriffe setzen Reinhold zufolge die Sprache voraus. Denn nur durch das sprachliche Bezugssystem haben mentale Bilder repräsentationalen Gehalt. Und nur wenn mentale Bilder repräsentationalen Gehalt haben, stellt sich allererst das Schematismus-Problem: Wie kann ein Begriff auf die ihm korrespondierenden Anschauungen angewandt werden? Reinholds Überlegungen zum Schematismus laufen also auf eine sprachliche, im Gegensatz zu Kants schematischer Hypotypose hinaus. ${ }^{14}$ Nach Reinhold fundiert die sprachliche Hypotypose die schematische

14 Kant führt den Begriff der Hypotypose oder Darstellung (exhibitio, subiecto sub adspectum) als Oberbegriff zu „Schema“ und „Symbol“ ein (Kant: KU, AA 05: 351.23-31). Die „schematische Hypotypose“ liefert eine direkte Anschauung für einen reinen Verstandesbegriff. Die „,symboli- 
Hypotypose. Dieser Gedanke findet sich weder bei Herder, Hamann noch bei Humboldt. Es handelt sich um Reinholds originellen Beitrag zu sprachphilosophischen Fundierung der Transzendentalphilosophie.

\section{Schlussbemerkung}

Zum Schluss möchte ich noch auf die Frage eingehen, was Kant zu Reinholds sprachphilosophischer Fundierung der Schematismuslehre gesagt haben könnte. Kants Einschätzung der sprachlichen Hypotypose hängt entscheidend davon ab, ob es nicht-begriffliche Wahrnehmungsinhalte gibt. Bekanntlich gibt es in der Kant-Forschung zwei Lager. Auf der einen Seite stehen Konzeptionalisten wie beispielsweise Ginsborg und McDowell, denen zufolge jede Gegenstandswahrnehmung Begriffe involviert. ${ }^{15}$ Für diese Lesart spricht, dass Kant Anschauungen ohne Begriffe für „blind“ hält. Auf der anderen Seite stehen Vertreter des nonconceptual content wie zum Beispiel Hanna und Allais. ${ }^{16}$ Dieser Fraktion zufolge ist der Inhalt der Wahrnehmung nicht-begrifflicher Natur. Wahrnehmungen haben also auch im vorbegrifflichen Zustand bereits Gehalt. Begriffe kommen erst ins Spiel, wenn der Wahrnehmungsgehalt in ein Wahrnehmungsurteil gegossen wird. Damit Reinholds These, wonach die schematische Hypotypose die sprachliche Hypotypose voraussetzt, zu Kant passt, muss die Transzendentalphilosophie im Sinne des Konzeptionalismus verstanden werden. Unter Voraussetzung des Konzeptionalismus sind die von der produktiven Einbildungskraft hervorgebrachten mentalen Bilder blind, das heißt inhaltsleer. Sie bedürfen des Begriffsapparats, um allererst Gehalt zu haben und um damit auch allererst mentale Bilder zu sein.

Die Schematismus-Lehre soll Kant zufolge das vermittelnde Dritte zwischen den blinden Anschauungen und den (leeren) Begriffen liefern. Reinhold zeigt, dass die Sprache für diese Vermittlung unerlässlich ist. Denn ohne die Sprache wären die mentalen Bilder inhaltsleer und die Zuordnung von mentalen Bildern

sche Hypotypose“ betrifft die indirekte Darstellung eines übersinnlichen Vernunftbegriffs (wie Gott und Seele). Da Vernunftbegriffe eigentlich nicht versinnlicht werden können, stimmen die Symbole mit dem betreffenden Vernunftbegriff „bloß analogisch“ zusammen (Kant: KU, AA 05: 352.08-352.12).

15 Ginsborg, Hannah: Was Kant a Nonconceptualist? In: Philosophical Studies, 137 (2008), 65- 77. McDowell, John: Mind and World. Cambridge (MA) 1994.

16 Allais, Lucy: Kant, Non-Conceptual Content and the Representation of Space. In: Journal of the History of Philosophy, 47 (2009), 383-413. Hanna, Robert: Kant and Nonconceptual Content. In: European Journal of Philosophy, 13 (2005), 248-290. 
zu Begriffen wäre deshalb willkürlich. Reinholds Theorie der sprachlichen Hypotypose stellt somit eine Ergänzung der konzeptionalistisch verstandenen Schematismuslehre dar. 
